\title{
Correlation of efficacy of cerebro placental ratio with adverse perinatal outcome in clinically suspected IUGR pregnancies
}

\author{
Geeta Singh $^{1}$, Neerja Gupta ${ }^{1 *}$, Sangeeta Singhal ${ }^{2}$, Pramod Kishor Sharma ${ }^{3}$
}

\begin{abstract}
${ }^{1}$ Department of Obstetrics and Gynecology, ${ }^{2}$ Department of Pediatrics, ${ }^{3}$ Department of Radio Diagnosis, Muzaffarnagar Medical College, Muzaffarnagar, Uttar Pradesh, India
\end{abstract}

Received: 19 February 2018

Accepted: 27 March 2018

\author{
*Correspondence: \\ Dr. Neerja Gupta, \\ E-mail: geetasingh2827@gmail.com
}

Copyright: () the author(s), publisher and licensee Medip Academy. This is an open-access article distributed under the terms of the Creative Commons Attribution Non-Commercial License, which permits unrestricted non-commercial use, distribution, and reproduction in any medium, provided the original work is properly cited.

\begin{abstract}
Background: IUGR is a most common and complex problem in modern obstetrics. Most commonly use methods to assess fetal condition are BPP and NST which are not sensitive for predicting better perinatal outcome. Present study was an effort to evaluate the role of ratio of pulsatility index (PI) of middle cerebral artery and umbilical artery which is called cerebro placental ratio as the most sensitive, specific and accurate predictor of adverse perinatal outcome in clinically suspected IUGR Pregnancies.

Methods: 50 clinically suspected IUGR Pregnancies attending antenatal clinics Muzaffarnagar Medical College and Hospital, Muzaffarnagar were subjected to Doppler ultrasound evaluation Doppler velocity wave form of umbilical artery and fetal middle cerebral artery were obtained. Pulsatility index ratio of MCA and umbilical artery (cerebro placental ratio) was evaluated in each case. Abnormal ratio is defined as CPR $<1.08$ considered as cut of value. Ratio was coo related clinically with perinatal outcome.

Results: Out of 50 antenatal cases, $63 \%$ neonates had birth weight $<2.5 \mathrm{~kg}$. There were 6 IUD'S and 44 live births, 9 neonates were admitted to NICU, 7 neonates had $5 \mathrm{~min}$. APGAR score $<7$ and 13 neonates were born by emergency CS. Of the 6 IUDS, 4 cases had reversal of blood flow umbilical artery and 2 cases had absent diastolic flow. In all cases of reversal Diastolic flow, IUD occurred within 7 days of diagnosis.

Conclusions: CPR is the most sensitive, specific and accurate parameter in prediction of adverse perinatal outcome and thus can help in decreasing perinatal mortality.
\end{abstract}

Keywords: Cerebro plecental ratio, Color doppler, IUGR, Pulsatility index

\section{INTRODUCTION}

IUGR is a common condition affecting about $10-15 \%$ of the general maternity population. ${ }^{1}$ It is associated with increased risk of perinatal mortality, morbidity and impaired neurological development. ${ }^{2}$ Before the advent of $\mathrm{U} / \mathrm{S}$, assessment of fetal growth during pregnancy was limited by measuring the uterine size, assessing the fetal size by palpation and looking at the infant after delivery but now-a-days, Doppler velocimetry provides a sensitive, non-invasive and safe method for surveillance of fetal hemodynamics and fetomaternal circulation. This modality is based on the premise that an insufficient uterine, placental or fetal circulations results in adverse perinatal outcome. UA Doppler is the most evaluated tool among non-invasive tests of fetal well-being. It serves as both a prognostic and diagnostic tool in assessment of IUGR fetus. ${ }^{3}$ An abnormal waveform of umbilical artery. (Absent or reversed end diastolic flow) has been demonstrated to predict fetal compromise. 12 days prior to acute fetal deterioration. ${ }^{4}$ In pregnancies with prolonged fetal hypoxia, there is redistribution of blood 
volume towards vital organs (i.e. heart, adrenal glands, spleen, brain and kidney), which causes vasodilation of the MCA, with an increase in diastolic flow hence decrease in its PI. ${ }^{5-8}$

Thus, in asymmetrical IUGR, there is a high UAPI and low MCA PI as a result CP ratio is lower than normal in growth retarded fetuses. $\mathrm{CP}$ ratio remains constant during last 10 weeks of gestation and so it is having a better diagnostic accuracy. ${ }^{9}$

The purpose of this study was to assess the efficacy of CP ratio to predict IUGR in clinically suspected IUGR cases and its correlation with perinatal outcome.

Objectives of this study were to evaluate the role of umbilical artery Doppler and MCA Doppler indices in growth restricted foetuses, to evaluate the significance of Doppler flow velocimetry in prediction of adverse perinatal outcome in clinically suspected IUGR pregnancy and to establish the role of color Doppler in management of IUGR pregnancy.

\section{METHODS}

Present study was a hospital based cross-sectional observational study conducted on clinically suspected IUGR pregnancies attending antenatal OPD of Obstetrics and Gynaecology Department of Muzaffarnagar Medical College and Hospital, Muzaffarnagar. This study was approved by the ethical committee of our Institution. Study size included 50 singleton pregnancies

\section{Inclusion criteria}

- Singleton pregnancies

- Fetal gestational age of 30-40 weeks with clinically suspected

- IUGR pregnancies

- Absence of preexisting maternal disease.

\section{Exclusion criteria}

- Multiple gestations

- Documented major congenital abnormality

\section{Methodology}

Study was done on antenatal women (30-40 weeks of gestational age) attending antenatal clinic of Muzaffarnagar Medical College and Hospital, Muzaffarnagar.

On the basis of detailed clinical history and examination, 50 clinically suspected IUGR cases were selected and subjected to ultrasound (including ultrasound biometry, assessment of amniotic fluid and placental maturity) and Doppler studies in the Department of Radio Diagnosis of
Muzaffarnagar Medical College and Hospital, Muzaffarnagar.

Examination was conducted on PHILIPS Clear VUE 650 with the help of multi frequency convex probe, two or three times.

- PI of both arteries were observed

- MCA: UA PI ratio was calculated

- Doppler study was considered abnormal if

a. UAPI $>95^{\text {th }}$ percentile for gestational age

b. MCA PI $<5^{\text {th }}$ percentile for gestational age

c. MCA/UA PI ratio $<1.08$

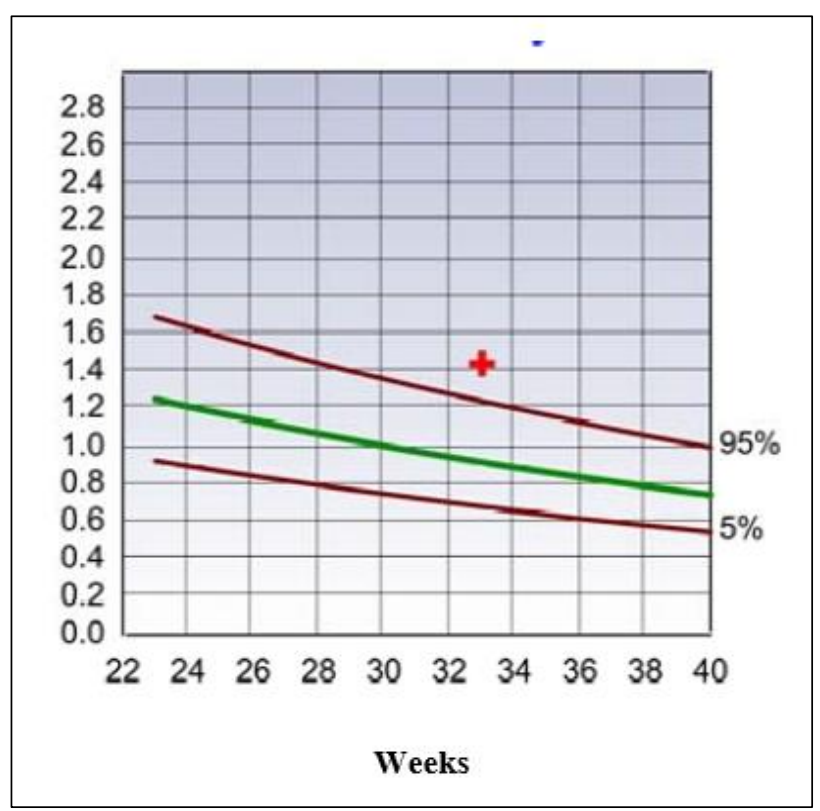

Figure 1: Distribution of normal UAPI with gestation.

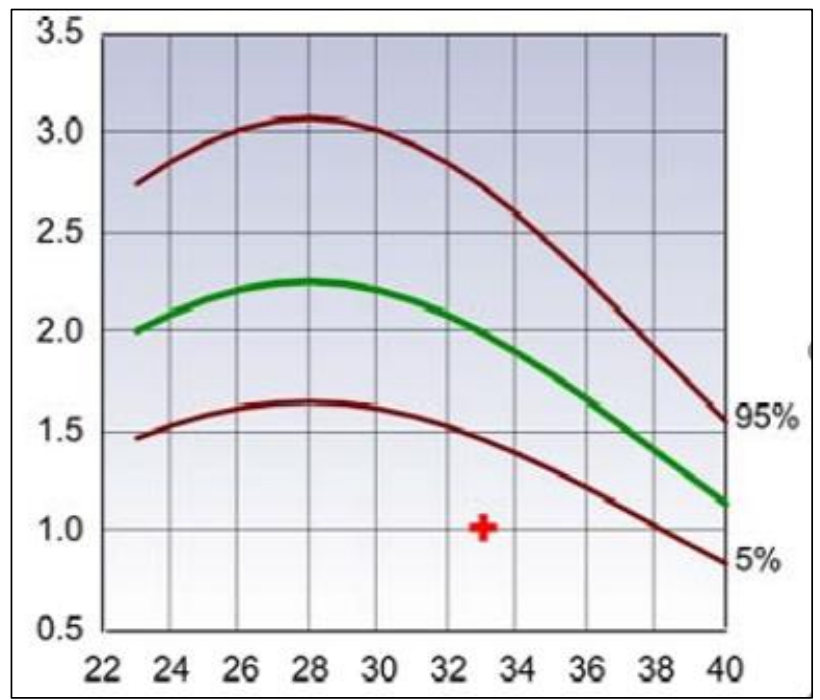

Figure 2: Distribution of normal MCA PI with gestation. 
Pulsataility indices for gestational age: the normal range is shown in $5^{\text {th }}, 50^{\text {th }}$ and $95^{\text {th }}$ percentiles

Adverse perinatal outcome includes intrauterine death, emergency LSCS, low APGAR score at $5 \mathrm{~min}$. <7, admission to NICU.

\section{RESULTS}

50 antenatal women between 30 to 40 weeks of gestation who were clinically suspected as having IUGR pregnancies based on clinical history of previous child with growth retardation, anemia, high blood pressure, reduce abdominal fundal height for gestational age (30 to 40 weeks) were evaluated using screening ultrasound and then Doppler velocity waveforms were obtained from umbilical artery and fetal middle cerebral artery. Out of 50 patients, $44 \%$ were between gestational age of 30 to 32 weeks, $20 \%$ were between 35 to 37 weeks and $36 \%$ were between 37 to 40 weeks (Table 1).

Table 1: Gestational age distribution in study group.

\begin{tabular}{|lll|}
\hline GA (Weeks) & Number & Percentage \\
\hline $30-32$ & 22 & 44 \\
\hline $35-37$ & 10 & 20 \\
\hline $37-40$ & 18 & 36 \\
\hline Total & 50 & 100 \\
\hline
\end{tabular}

$62 \%$ of patients were primi gravida and $38 \%$ were multigravida (Table 2).

Table 2: Parity distribution of study group.

\begin{tabular}{|lll|}
\hline Parity & Number. of cases & Percentage \\
\hline Primi & 31 & 62 \\
\hline Multi & 19 & 38 \\
\hline
\end{tabular}

Out of 50 patients $68 \%$ had PIH, $22 \%$ had anemia, $4 \%$ had $\mathrm{BOH}$ and $2 \%$ had diabetes mellitus and $4 \%$ had chronic renal disease at first Doppler ultrasound (Table 3).

Table 3: Maternal complications of study group.

\begin{tabular}{|lll|}
\hline Complication & No. of cases & Percentage \\
\hline PIH & 34 & 68 \\
\hline Anemia & 11 & 22 \\
\hline BOH & 2 & 4 \\
\hline DM & 1 & 2 \\
\hline Chronic renal disease & 2 & 4 \\
\hline
\end{tabular}

Table 4: Distribution characteristics of placental maturity.

\begin{tabular}{|lll|}
\hline Grade & No. of cases & Percentage \\
\hline Grade II & 11 & 22 \\
\hline Grade III & 39 & 78 \\
\hline
\end{tabular}

On ultrasound $78 \%$ of cases show grade 3 placental maturities, $22 \%$ had grade 2 placental maturity (Table 4). $76 \%$ had oligohydroannios and $24 \%$ had normal amniotic fluid (Table 5).

Table 5: Amniotic fluid distribution in study group.

\begin{tabular}{|lll|}
\hline & No. of cases & Percentage \\
\hline Normal & 12 & 24 \\
\hline Oligohydroamnios & 38 & 76 \\
\hline
\end{tabular}

Out of 50 antenatal cases, $63 \%$ neonates had birth weight $<2.5 \mathrm{~kg}$. There were 6 IUD'S and 44 live births, 9 neonates were admitted to NICU, 7 neonates had 5 min. APGAR score $<7$ and 13 neonates were born by emergency CS (Table 6).

Table 6: Adverse perinatal outcome.

\begin{tabular}{|lll|}
\hline $\begin{array}{l}\text { Adverse perinatal } \\
\text { outcome indicator }\end{array}$ & $\begin{array}{l}\text { No. of } \\
\text { cases }\end{array}$ & Percentage \\
\hline IUD & 6 & 12 \\
\hline Emergency LSCS & 13 & 26 \\
\hline Low APGAR Score & 7 & 14 \\
\hline Admission To NICU & 9 & 18 \\
\hline
\end{tabular}

Out of 6 IUD's, 4 cases had reversal of diastolic flow and 2 had absent diastolic flow. In all cases of reversal of diastolic flow, IUD of the fetus occurred within 7 days of diagnosis- S/O 100\% mortality and all the 3 cases were less than 32 weeks. In the present study, sensitivity and specificity of cerebro-placental ratio was $93.2 \%$ and $89 \%$ respectively which was more than sensitivity and specificity of UA PI and MCA PI (Table 7).

Table 7: Performance characteristics of Doppler indices.

\begin{tabular}{|lll|}
\hline Doppler Index & Sensitivity & Specificity \\
\hline UA PI & $88.6 \%$ & $81 \%$ \\
\hline MCA PI & $80.0 \%$ & $70 \%$ \\
\hline CPR (MCAPI/UAPI) & $93.2 \%$ & $89 \%$ \\
\hline
\end{tabular}

The diagnostic accuracy in prediction of adverse in clinically suspected IUGR cases $\mathrm{CP}$ ratio has the diagnostic accuracy of $91.6 \%$, and MCA PI was $72.6 \%$ and UA PI was $79.6 \%$ (Table 8 ).

Table 8: Diagnostic accuracies of doppler indices.

\begin{tabular}{|ll|}
\hline Doppler index & Diagnostic accuracy \\
\hline UA PI & $79.6 \%$ \\
\hline MCA PI & $72.6 \%$ \\
\hline MCA/UA PI ratio & $91.6 \%$ \\
\hline
\end{tabular}

Present study results in evaluating the usefulness of MCA PI /UA PI ratio as strong predictor of adverse outcome in IUGR and thus it helps in decreasing perinatal mortality and morbidity. 


\section{DISCUSSION}

IUGR is a pathological condition characterized by fetal weight $<10^{\text {th }}$ percentile for that gestational age. ${ }^{10}$ It is strongly related to uteroplacental insufficiency.

In normal pregnancy, the indices PI decrease with advancing gestation in UA. But in IUGR first there is decrease in diastolic flow of UA due to increase in the resistant that occurs in small Artery and arterioles of tertiary Villi. ${ }^{11}$ This raises the S/D ratio and PI of umbilical Artery. As the placental insufficiency Worsen the diastolic flow decreases, then become absent and later reverse flow pattern noted in umbilical artery. ${ }^{12,13}$

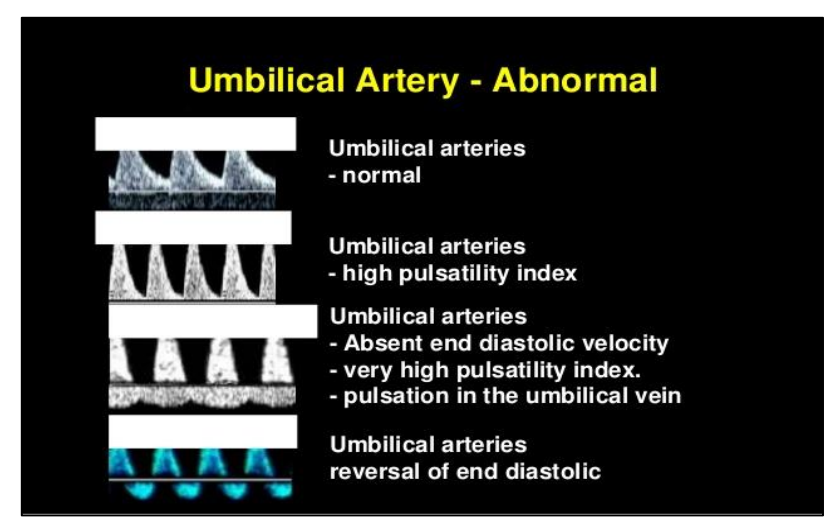

Figure 3: Umbilical artery waveforms.

Fetal MCA is a low resistance circulation throughout pregnancy and account of $7 \%$ of cardiac output. In fetal hypoxia and ischemia, increase in diastolic flow with decreased pulsatility. ${ }^{14,15}$ Index shows the brain sparing taking place in compromised foetuses. ${ }^{16}$ Fetal hypoxia results into adverse fetal outcome.

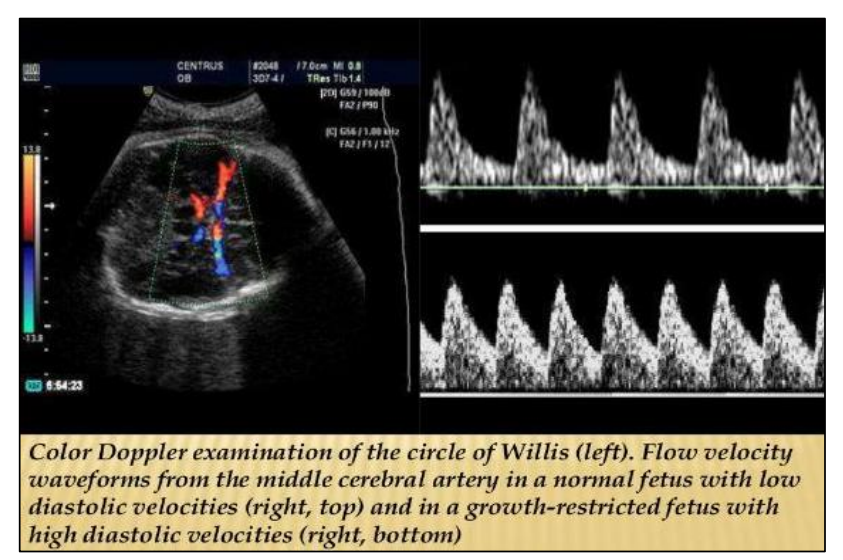

Figure 4: Middle cerebral artery waveforms.

MCA/UA PI ratio reflects not only the circulatory insuffiency of the umbilical velocimetry of the placenta manifested by alteration in umbilical S/D ratio but also the adaptive changes resulting in modification of the middle cerebral artery S/D ratio. ${ }^{16} \mathrm{MCA} / \mathrm{UA}$ PI ratio reflects both placental status and fetal response. So, this gives additional information. Doppler velocimetry is an important non-invasive technique for fetomaternal surveillance in high risks pregnancies.

In the present study diagnostic accuracy of MCA/UA PI was $91.6 \%$, and UA PI was $79.6 \%$ and MCA PI $72.6 \%$ for adverse perinatal outcome. The values are comparable with Gramillini et al and Bano et al. ${ }^{16,17}$

We choose 50 clinically suspected IUGR pregnancies and majority of the neonates were between 1.5 to $2 \mathrm{~kg}$ birth weights; we select incidences of IUD, Emergency, Cesarean section for fetal distress, NICU admission for complication of low APGAR score as outcome variables in concurrence with previous studies.

$\mathrm{CP}$ ratio has high sensitivity, specificity and diagnostic accuracy in predicting adverse perinatal outcome. This result is comparable with Lakhar et al, Fong KW et al, Bahado et al. ${ }^{15,18,19}$

Present study evaluates the efficacy of umbilical artery and cerebro placental ratio in prediction of adverse perinatal outcome in IUGR and indicates that both abnormal umbilical artery and $\mathrm{CP}$ ratio are strong predictors of adverse outcome in IUGR.

\section{CONCLUSION}

In fetal growth restriction, $\mathrm{CP}$ ratio reflects both circulatory insufficiencies of placenta and also adaptive changes that occurs in middle cerebral artery, so it appears to be a valuable non-invasive modality for feto maternal surveillance in IUGR.

Abnormal CP ratio is a better predictor of adverse perinatal outcome in IUGR. Doppler ultrasound plays a significant role in the management of growth restricted fetuses by early identification and thus helps in line of management, obstetrical surveillance.

Hence, Doppler ultrasound especially CP ratio (MCA/UI PI Ratio) should be an integral component of routine evaluation of a suspected IUGR pregnancies and thereby improving adverse perinatal outcome.

Funding: No funding sources Conflict of interest: None declared

Ethical approval: The study was approved by the Institutional Ethics Committee

\section{REFERENCES}

1. Gardosi J. Clinical strategies for improving the detection of fetal growth restrictions. Clin Perinatol. 2011;38:21-31.

2. Shand AW, Buckle JH, Nathan E, Dickinson JE, French NP. Small for gestational age, preterm infants and relationship of abnormal artery Doppler blood 
flow to perinatal mortality and neuro development outcome. Aust N Z J Obstet Gynecol. 2009;49(1).

3. Figueras F, Gratacos E. Update on the diagnosis and classification of fetal growth restriction and proposal of a stage-based management protocol. Fetal Diagn Ther. 2014;36:86-98.

4. Bekedam DJ, Visser GH, Vander Zee AG, Snijders RJ, Poelmann-Weesjes G. Abnormal velocity waveforms of umbilical artery in growth-retarded fetuses; relationship to antepartum late $\mathrm{hr}$ decelerations and outcome. Early Hum Dec. 1990;24:79-89.

5. Campbell S, Vyas S, Nicolaides KH. Doppler investigation of fetal circulation. J Perinat Med. 1991;19:21-6.

6. Mari G, Uerpairjkit B, Abuhamad AZ, Copel JA. Adrenal artery velocity waveforms in appropriate and small for gestation fetuses. Ultrasound Obstet. Gynecol. 1996;8:82-6.

7. Abuhamad AZ, Mari G, Bogdan D, Evansep. Doppler flow velocimetry of the splenic artery in human fetus; is it a matter of chronic hypoxia? Am J Obstet Gynaecol, 172, 1995, 820-825.

8. Hecherk, Campbell S, Doyle P, Herrington K, Nicolaides KM. Assessment of fetal compromised by Doppler ultrasound investigation of the fetal circulation: Arterial, intracranial and venus blood flow velocity studies. Circ. 1995;91:129-38.

9. Giles WB, Trudinger BJ, Baird PJ. Fetal umbilical flow velocity waveform and placental resistance pathological correlation. $\mathrm{Br} \mathrm{J}$ Obstet Gynaecol 1985;92:31-8.

10. Battaalia FC, Lubchenko LO. A practical classification of newborn infants by weight and gestational age. J Pediatr. 1967;71:159-62.

11. Piazze J, Padula F, Cerekja A, Cosmi EV, Anceschi MM. Prognostic value of umbilical-middle cerebral artery pulsatility index ratio in fetuses with growth restriction. Int J Gynaecol Obstet. 2005;91(3):233-7.

12. Erlan AK, He JP, Tanriverdi HA, Hendrick J, Linvach HG, Schmidt W. Comparison of perinatal outcome in fetuses with reverse or absent diastolic flow in the umbilical artery and/or fetal descending aorta. J Perinat Med. 2003;31(4):307-12.
13. Gerber S, Hohlfeld P, Viquerat F, Tolsa JF, Vial Y. Intrauterine growth restriction and absent or reverse end-diastolic blood flow in umbilical artery (Doppler class II or III): A retrospective study of short- and long-term fetal morbidity and mortality. Eur J Obstet Gynecol Reprod Biol. 2006;126(1):20-6.

14. Strigini FA, De Luca G, Lencioni G, Scida P, Giusti G, Genazzani AR. Middle cerebral artery velocimetry: different clinical relevance depending on umbilical velocimetry. Obstet Gynecol. 1997;90(6):953-7.

15. Lakhkar BN, Rajagopal KV, Gourisankar PT. Doppler prediction of adverse perinatal outcome in PIH and IUGR. IJRI. 2006;16(1):109-16.

16. Gramellini D, Folli MC, Raboni S, Vadora E, Merialdi A. Cerebral umbilical Doppler ratio as a predictor of adverse perinatal outcome. Cerebral and umbilical ratio. Obstet Gynecol. 1992;79:416-20.

17. Bano S, Chaudhary V, Pande S, Mehta VL, Sharma AK. Color doppler evaluation of cerebral-umbilical pulsatility ratio and its usefulness in the diagnosis of intrauterine growth retardation and prediction of adverse perinatal outcome. Indian J Radiol Imaging 2010;20:20-5.

18. Fong $\mathrm{KW}$, Ohlsson A, Hannah ME, Grisaru S, Kingdom J, Cohen H, et al. Prediction of perinatal outcome in fetuses suspected to have intrauterine growth restriction: Doppler US study of fetal cerebral, renal, and umbilical arteries. Radiol. 1999;213(3):681-9.

19. Bahado-Singh RO, Kovanci E, Jeffres A, Oz U, Deren O, Copel J, et al. The Doppler cerebroplacental ratio and perinatal outcome in intrauterine growth restriction. Am J Obstet Gynecol. 1999;180:750-6.

Cite this article as: Singh G, Gupta N, Singhal S, Sharma PK. Correlation of efficacy of cerebro placental ratio with adverse perinatal outcome in clinically suspected IUGR pregnancies. Int J Reprod Contracept Obstet Gynecol 2018;7:1808-12. 